\title{
Rehabilitation of a water distribution system with diffused water losses
}

\author{
G. Festa ${ }^{1}$, D. Verde ${ }^{2}$ \& R. Magini ${ }^{3}$ \\ ${ }^{1}$ Civil Engineer, Italy \\ ${ }^{2}$ AcquaLatina S.p.A, Italy \\ ${ }^{3}$ Department of Hydraulics, Transportation \& Roads, \\ Sapienza', University of Rome, Italy
}

\begin{abstract}
In this paper a rehabilitation plan for the water distribution system (WDS) of the small town of Fondi, located $100 \mathrm{~km}$ south of Rome (Italy), is presented. In particular the most concerning problem of this WDS is the high level of leakages, which are estimated to more than $60 \%$ of the supplied water volume. Due to the old age and very bad conditions of the pipes, the leakages are uniformly spread all over the network. For this reason the most effective strategy to reduce water losses can be only the renovation of the network. In particular the rehabilitation plan provides where to intervene (which pipes), how (pipe substitution with a defined diameter) and when (at a fixed moment in a time horizon). The optimal scheduling is achieved by means of the multi objective genetic algorithm NSGAII aimed to minimize the water losses and the costs of the interventions, and the pressure distribution variance. The applied methodology uses a 'pressure driven' hydraulic model of the WDS, which considers the leakages and locates them. The model calibration is carried out using a genetic algorithm (GA).
\end{abstract}

Keywords: water distribution systems, leakages, rehabilitation, genetic algorithms, NSGAII.

\section{Introduction}

Water losses occurring in water distribution systems (WDS) are often the main concern for Water Utility companies. Actually in some regions of Italy leakages can represent up to $70 \%$ of the water volume supplied to the network. This huge 
lost water volume is a serious economic damage for the companies. It causes high costs for water pumping and treatment, the necessity of investments in systems to expand their capacity, and/or in searching for new water sources. Moreover, in many regions, nowadays the biggest concern is the impoverishment of the water resources, due to pollution, to droughts, and to the increasing demand. Therefore is of vital importance to save the available water resources.

Usually many WDS are aged, poorly maintained and consequently present a progressive deterioration of the pipes conditions, which determines high percentages of water losses. In these cases the largest part of the water losses are diffused. Indeed the leakages are spread over the network, especially at junctions or fittings or at little cracks on the pipes. For these reasons the leakages research techniques, carried out by noisy-loggers, electro-acoustic instruments or correlators are not effective or economically efficient. Whereas, in a short time horizon good results can be obtained optimizing pressures by installing dynamic reduction valves. Obviously, the most effective solution in a mid-long time horizon can be the renewal of the WDS.

The rehabilitation problem attempts to identify the pipes that should be replaced or cleaned and/or relined and the timing of those actions in a defined time horizon. In particular the aim of a rehabilitation plan is to find the answers to the questions of where to intervene, how and when (Alvisi and Franchini [1]). This issue is usually tackled as a multi-objective optimization problem, in which the objective function is represented by the performance of the network and the costs of the rehabilitation (Halhal et al. [2]). The performance index varies depending on the specific WDS and the authors. WDS reliability, frequently used (Dandy and Engelhardt [3]) is defined as the capability to satisfy the demand together with adequate pressure head and water quality. The reliability of the WDS decline when water demand or pipes roughness increase or pipes failure occurs. Common objectives functions are the not delivered water volumes or the numbers of customer affected by interruptions due to pipes bursts (Engelhardt [4]). Such a condition implies the authors to develop models able to generate pipe breaks, $[1,5]$. Another objective to be pursued is the increase of the network efficiency through the reduction of water losses. Depending on the performance index, the costs to be minimized, other than capital costs for pipes replacement, cleaning and relining, can include pipes breaks repairs and water supply (pumping and treatments). The limited funds available constrain the invested annual budget and increase the importance of interventions time scheduling. Genetic algorithms are by far the most used optimization approach.

In this work a rehabilitation plan for the WDS of Fondi, a small town located $100 \mathrm{~km}$ south of Rome, is presented. The network is characterized by a percentage of water losses exceeding $60 \%$ of the supplied water volume. Due to this large amount of water losses and to their uniform distribution all over the network, the rehabilitation plan is firstly aimed to the minimization of the leakages. In the procedure only pipes replacement is considered as possible intervention. Because of the high cost per cubic meter of the water, due to the pumping, the energy costs are added to the costs of pipes replacements in the overall costs minimization. Moreover, to guarantee a uniform spatial distribution 
of the nodal pressures with the new configuration of pipes, the standard deviation of nodal pressures is also introduced. This multi-objective optimization is carried out by the NSGAII genetic algorithm.

The present rehabilitation approach, mainly focused on water losses reduction, embeds a pressure driven hydraulic model, which guarantees a more realistic simulation of the leakages. A specific procedure used for the model calibration allows one to determine the distribution of the water losses in the network. Such leakages distribution implies the optimization algorithm to indicate which pipes to replace first. The model calibration procedure is achieved through a genetic algorithm.

\section{Model calibration and leakages location}

\subsection{Fondi's WDS description}

Fondi is supplied by a large tank located $4 \mathrm{~km}$ far from the city centre. The water is delivered to the distribution network through a steel main pipe of $700 \mathrm{~mm}$ diameter, which is split in two mains of $350 \mathrm{~mm}$ and $500 \mathrm{~mm}$ diameter respectively. Both the two mains, whose length is almost $4 \mathrm{~km}$, get then directly into the distribution network. Both the main pipes are installed with pressure reduction valves programmed with two different patterns (day and night). The water is pumped to the tank from a $65 \mathrm{~m}$ further down spring.

The $40 \mathrm{~km}$ long distribution network, with pipes diameters ranging from $32 \mathrm{~mm}$ to $500 \mathrm{~mm}$, is quite old (up to 60-70 years). In particular, the city centre pipes are almost made of cast iron (45\% of whole network) or steel and concrete (14\%). The PE, PEAD, PVC pipes cover the $38 \%$ of the network and are the most recent. The WDS supplies water to 9800 customers, mainly residential, corresponding to about 25000 inhabitants. An accurate study of the users bills of the past five years allowed to estimate the average consumption of 216 litres per person per day, corresponding to the average flow of $62.51 / \mathrm{s}$. Flow measurements recorded between the $17^{\text {th }}$ and the $25^{\text {th }}$ of January 2008 show an average flow supplied of $153.81 / \mathrm{s}$. Then leakages can be estimated to about $901 / \mathrm{s}$ (60\% of the supplied water volume.)

\subsection{Model calibration}

For the high percentage of water losses, the hydraulic model of the WDS should simulate them realistically. Depending the leakages on pressure, therefore the hydraulic model follows a pressure driven approach. Leakages are concentrated at the nodes using the following formula:

$$
L_{i}=k_{i} \cdot p_{i}^{n}=\left[c_{z} \cdot \frac{1}{2} \sum_{j}^{N i} l_{i j}\right] \cdot p_{i}^{n}
$$

where the leakage $L_{i}$ in the $i^{\text {th }}$ node is proportional to its pressure $p_{i}$ through the emitter coefficient $k_{i}$ and the exponent $n$. Each pipe $j^{\text {th }}$ connected to the node $i^{t h}$ contributes to the value of the emitter coefficient $k_{i}$ proportionally to its length $l_{i j}$. Moreover all nodes belonging to a same defined zone $z$, in which the network is 
previously divided, have the same water losses coefficient $c_{z}$. The exponent $\mathrm{n}$ could range from 0.5 to 2.5 . The most common values in the technical literature are 1.15-1.18 (Fantozzi and Lambert [6] and Germanopoulos [7]).

The model calibration is aimed to estimate the vector of pipes roughness coefficients $\underline{\varepsilon}$, and the water losses coefficients $\underline{c}$. To this purpose the network pipes are previously grouped for different roughness coefficient considering the pipes materials and the network topology. A similar zoning is carried out also for the nodes and their water loss coefficients $\underline{c}$. It is important to underline how the latter division of the network in zones and subsequent estimation of water losses coefficients determines the spatial distribution of the leakages. The procedure calibration estimates $\underline{\varepsilon}$ and $\underline{c}$ minimizing the following objective function $o . f$ :

$$
\text { o.f. }=\sum_{i=1}^{T} \sum_{i=1}^{N p} \frac{\left[p_{i}^{\text {meas }}-p_{i}^{c l c}(\underline{c}, \underline{\varepsilon})\right]^{2}}{\left(p_{i}^{\text {meas }}\right)^{2}}+\sum_{t=1}^{T} \sum_{j=1}^{N q} \frac{\left[q_{j}^{\text {meas }}-q_{j}^{c l c}(\underline{c}, \underline{\varepsilon})\right]^{2}}{\left(q_{i}^{\text {meas }}\right)^{2}}
$$

sum of the square differences between $\mathrm{N}_{\mathrm{p}}$ nodal pressures $p_{i}$ and $\mathrm{N}_{\mathrm{q}}$ pipe flows $q_{j}$ measured and calculated at different $\mathrm{T}$ time intervals in the 24 hours.

The pressure is measured in $\mathrm{N}_{\mathrm{p}}=6$ different nodes, uniformly distributed in the network. $\mathrm{N}_{\mathrm{q}}=4$ is the number of monitored pipe flows. $\mathrm{T}=6$ different time intervals are considered. The pipes of the WDS are grouped in 6 different zones related to the roughness coefficients $\varepsilon$. The WDS is also divided in 8 different areas $z$, each one with its water losses coefficient $c_{z}$. The number of unknowns of the optimization problem is 14 .

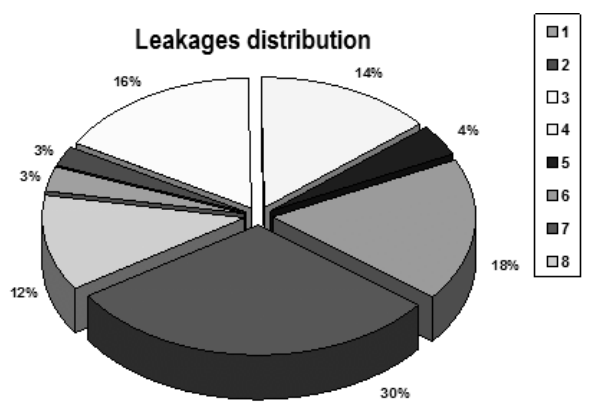

Figure 1: Water losses distribution per zone.

The optimization is carried out by a genetic algorithm (GA) coupled with the WDS hydraulic model, implemented by Epanet2. In order to address the problem toward the correct solution, inequality constrains on the error between the measured and calculated flows at the two main pipe are considered (mass continuity condition). The domain of $\underline{c}$ is set between $1 \times 10^{-5}$ and $51 \times 10^{-5} \mathrm{l} / \mathrm{s} / \mathrm{m}^{\mathrm{n}+1}$, while $\underline{\varepsilon}$ ranges from 80 to 160 (Hazen-William coefficient.) The $n$ exponent is equal to 1.15 . The minimum of the o.f. is obtained for $\varepsilon$ ranging from 120 to 150 and for the following water losses coefficients $c$ :

\begin{tabular}{|c|c|c|c|c|c|c|c|}
\hline $\mathrm{c}_{1}$ & $\mathrm{c}_{2}$ & $\mathrm{c}_{3}$ & $\mathrm{c}_{4}$ & $\mathrm{c}_{5}$ & $\mathrm{c}_{6}$ & $\mathrm{c}_{7}$ & $\mathrm{c}_{8}$ \\
\hline $1.10^{-5}$ & $1.10^{-5}$ & $9.10^{-5}$ & $3.10^{-5}$ & $1.10^{-5}$ & $7.10^{-5}$ & $35.10^{-5}$ & $25.10^{-5}$ \\
\hline
\end{tabular}


From these values results that some zones are much more affected by leakages than others; namely the $7^{\text {th }}$ zone (city centre) and $6^{\text {th }}$ zone are responsible for almost $50 \%$ of the global leakages, Figure 1 . While the $8^{\text {th }}$ area, together with $7^{\text {th }}$, has the biggest losses per km of pipes, Figure 2 .

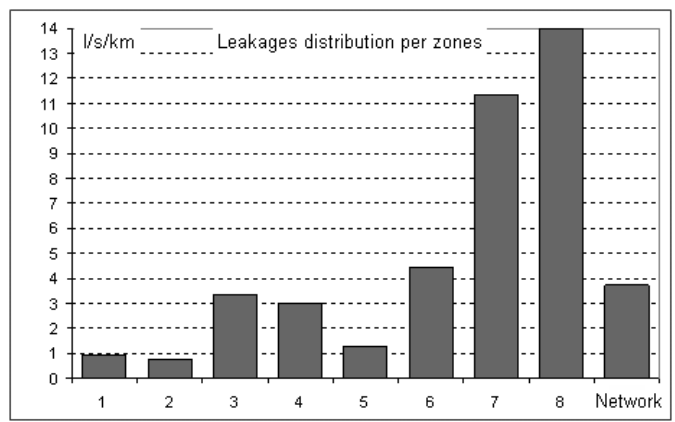

Figure 2: Water losses: $1 / \mathrm{s}$ per $\mathrm{km}$ of pipes in each zone.

\section{Rehabilitation plan}

Fondi's water distribution network rehabilitation is realized by planning a 5 years series of intervenes on pipes (substitution). The maximum number of replacement per year is fixed in order to remain under a threshold (3 intervenes per year), so that 15 intervenes can be performed at a max. A constrain related to the maximum available budget per year is also introduced $(300,000 €)$. Both the constrains are strictly demanded by the Water Utility.

The decision variable is represented by a matrix with rows equivalent in number to the maximum series of intervenes available during the five years term, and columns representing the pipe's id, type of rehabilitation and year respectively. The decision variable is created generating each intervene (row) by considering that each pipe can be entered the matrix only one time. That's implies an increase of the conditioned probability related to an un-chosen pipe once the implementation of the matrix goes on.

The substitution of the pipe is the only type of intervene considered. The new pipe can be of the immediately smaller, or immediately larger or of the same commercial diameter of the substituted pipe. Each time the pipes roughness is redefined at starting level (150 of Hazen William coefficient). For each pipe substitution, the consequent reduction of leakages is accomplished by reducing the emitter coefficients of the nodes at its ends. The reduction, equal to $90 \%$, is referred only to the part of the coefficient concerning the pipe at issue. The value of $90 \%$ comes from the hypothesis that $10 \%$ of water losses is unavoidable and acceptable threshold for an efficient WDS.

The overall cost associated with the substitution (depending on the diameters) must remain under the annual budget threshold. In order to avoid the algorithm to fall into an unclosed loop a critical number of cycles, aimed at creating the compatible intervene, is introduced. Once reached such a threshold, the row is annulled (no intervene available in that year). 
Hydraulic constrains are used in order to impose the pressure head at each node always larger than a minimum fixed value $(20 \mathrm{~m})$.

The $o . f$. is represented by a three components vector.

The first component is a measure of the global cost related to the performed intervenes and the global volume of water pumped. Such a function depends both on the type of rehabilitation $\left(C_{R}\right.$ costs are non-linearly proportional to the diameters), on the global leakage $\left(\mathrm{C}_{\mathrm{V}}\right.$ energy costs for pumping) and on time (a interest index $r$ is introduced.) The component is described by the following equation:

$$
\text { Min } C_{G}=\sum_{i=1}^{5}(1+r)^{i}\left(C_{R, i}+C_{V, i}\right)
$$

The second component is the flow supplied to the network at the end of the $5^{\text {th }}$ year. Such a component helps defining the global condition reached by the network in terms of leakage independently of the rehabilitation costs.

$$
\text { Min } q_{5}
$$

The third component is represented by the standard deviation of the pressure head $\sigma(p)$ calculated at the $5^{\text {th }}$ year. Such a variable justifies the replacement of a pipe with a bigger diameter. Indeed the previous components are usually badly affected by this kind of substitution . A larger set of diameters helps the network in reaching a more even pressure distribution.

$$
\text { Min } \sigma(p)
$$

The problem to be solved can be expressed as follows:

$$
\text { M.O. }\left\{\begin{array}{l}
\operatorname{Min} \quad F\left(C_{G}, q_{5}, \sigma(p)\right) \\
\forall i, j, X_{\text {link }, i} \neq X_{\text {link }, j} \\
C_{R, j} \leq C_{R, \max }, j=1 \ldots N_{\text {years }} \\
h_{j} \geq h_{\min }, j=1 \ldots N_{\text {nodes }}
\end{array}\right.
$$

The NSGA II algorithm is used to determine the Pareto frontier. It is set with an unvarying number of individuals composing the population (1000 decision variables) and is performed for a total amount of 50 cycles (generations). The reproduction is realized by mixing up two solutions distributed at the opposite in respect to the rank order. By each couple just one subsequent solution can be generated. Mutation probability is fixed at $10 \%$.

\section{Results}

The coverage reached by the problem solutions in the given domain can be summarized in the following way:

The resulting supplied flow at the fifth year is spread between the starting level (153.8 1/s) and a minima of 1371/s.

The total amount of the rehabilitation cost is variable between 100,000 and $600,000 €$ (its available domain could be up to $1,500,000 €$ ). 
The value of the maximum standard deviation related to the total head is set between 8.5 and $10.5 \mathrm{~m}$ (Figure 3). Such a limited result in terms of leakages reduction and of total available budget exploited is mainly due to the limited number of intervenes per year (three) imposed by the Water Utility.

Each set of solutions optimizing the level reached by the function first two components (cost of rehabilitation and flow) is characterized by an increased value of the third one (standard deviation of the total head). The problem can be in such a way considered a true multi-objective one (Figure 4).

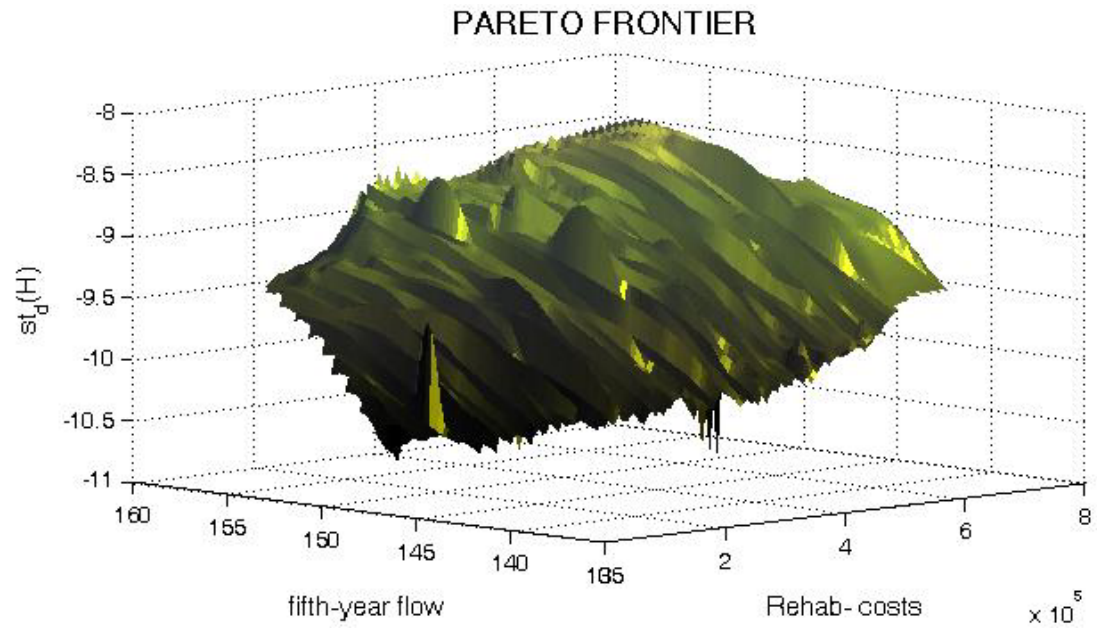

Figure 3: $\quad$ Pareto frontier.

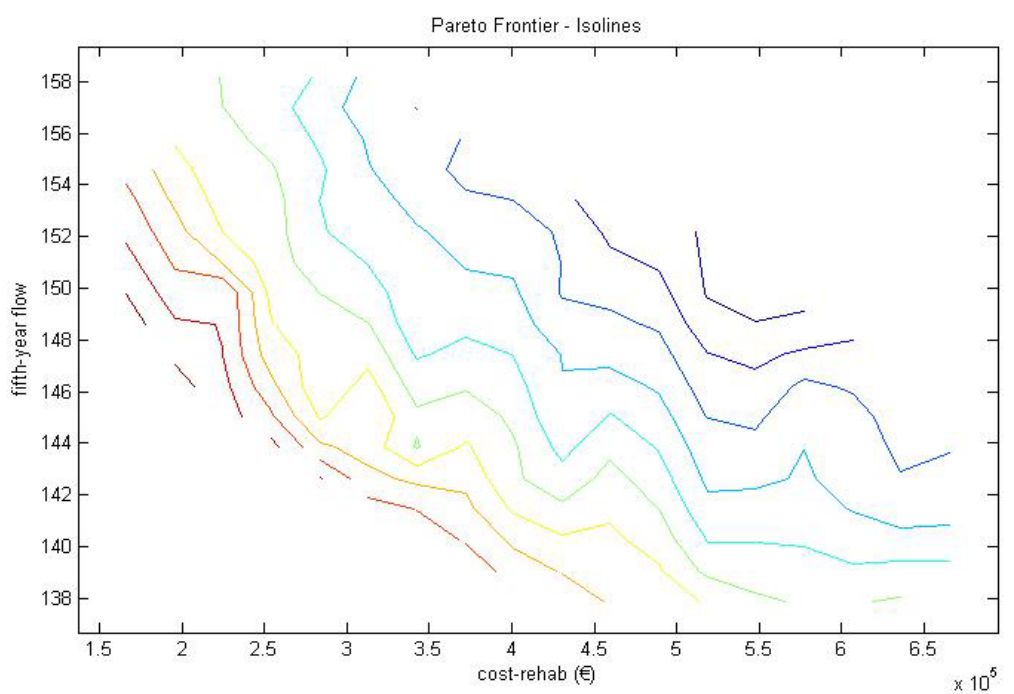

Figure 4: $\quad$ Contour plot: Costs vs Leakages for different values of $\sigma(p)$. 
The results underline that the number of the intervenes is centered on defined pipes (Figure 5). In particular pipes 22, 68-69, 79-81, 90, 100, 110 are characterized by the highest frequencies. It is interesting to point out how most of the previous rehabilitated pipes belong to the main leaking zones, $7^{\text {th }}$ and $8^{\text {th }}$ (Figure 6).

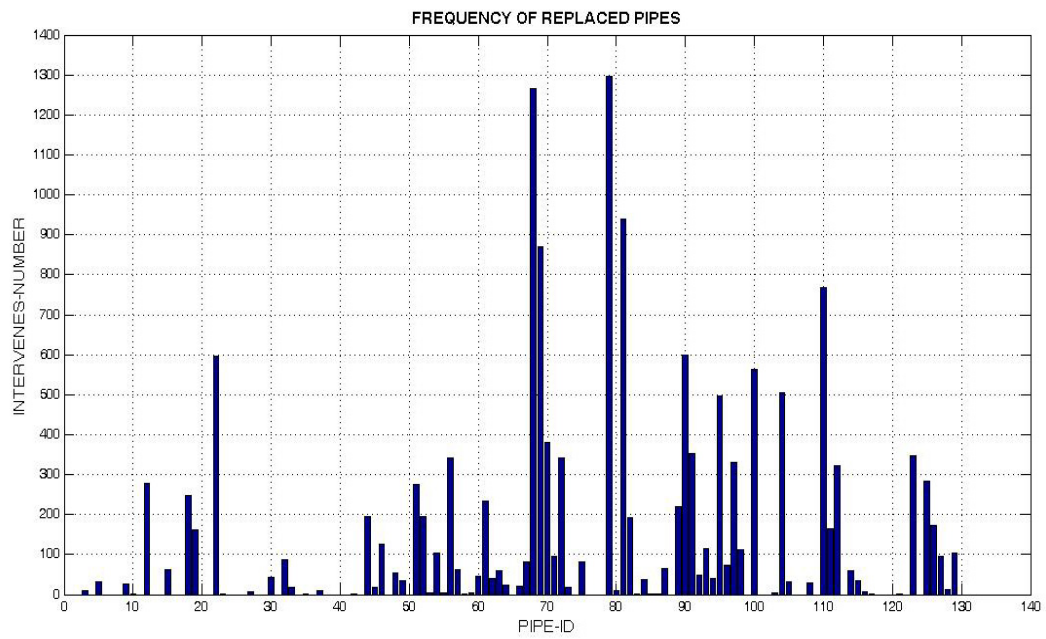

Figure 5: $\quad$ Frequency of pipes substitutions.

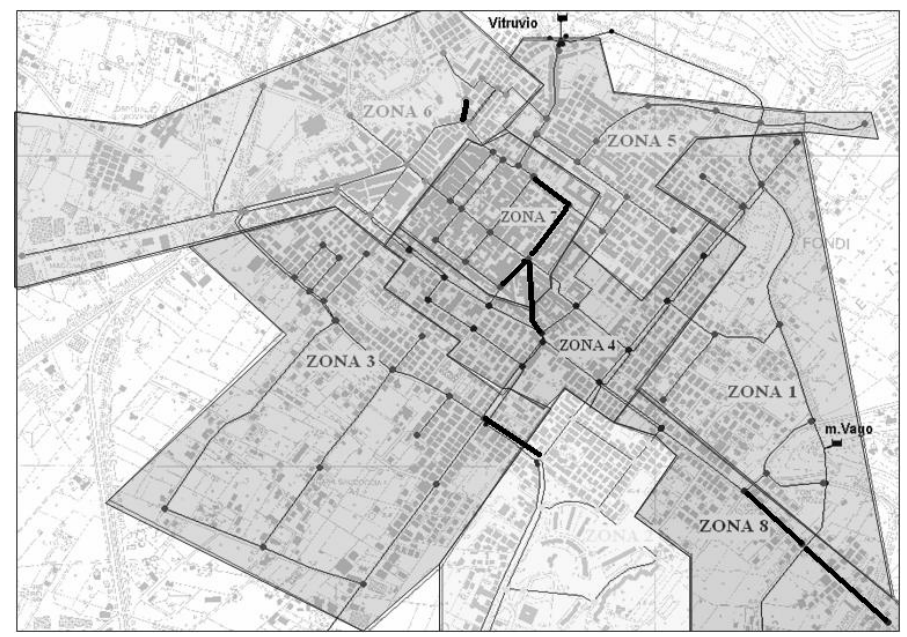

Figure 6: $\quad$ Position of the 8 pipes with the largest frequency of substitution. 


\section{Conclusions}

This paper present the rehabilitation plan for the WDS of Fondi, a town located $100 \mathrm{~km}$ south of Rome (Italy), which is affected by a high level of leakages, more than $60 \%$ of the supplied water volume. Due to the old age and very bad conditions of the pipes, the leakages are uniformly diffused and the most effective strategy for their reduction is the renovation of the network. In particular the rehabilitation plan provides where to intervene (which pipes), how (pipe substitution with a defined diameter) and when (at a fixed moment in a time horizon). The optimal scheduling is achieved by means of the multi objective genetic algorithm NSGAII aimed to minimize the water losses, the costs of the interventions, and the pressure distribution variance. The applied methodology uses a 'pressure driven' hydraulic model of the WDS, which considers the leakages and locates them. The model calibration, carried out using a GA., allowed, moreover, to divide the network in 8 zones, characterized by different percentage of water losses.

The results show that the supplied flow can be reduced up to the $12 \%$, which means a maximum water loss reduction of about the $20 \%$. This moderate reduction of leakages is mainly due to the limited number of intervenes per year imposed by the Water Utility.

\section{Acknowledgements}

The authors acknowledge the funding support from AcquaLatina Spa and are particularly grateful to Dr. Ennio Cima and Dr. Stefano Giulioli for their assistance.

\section{References}

[1] Alvisi S. \& Franchini M., Rehabilitation scheduling of water distribution systems based on multi objective genetic algorithms. Proc. of the $8^{\text {th }}$ International Conference in Computer and Control in Water Industry, 2005, Exeter, UK.

[2] Hahal D., Walters, G.A., Ouzar, D., Savic, D.A., Water network rehabilitation with structured messy genetic algorithm, Journal of Water Resources Planning and Management, ASCE, 123(3), 1997

[3] Dandy G.C. \& Engelhardt M.O., The optimal scheduling of water pipe replacement using genetic algorithms, Journal of Water Resources Planning and Management, ASCE, pp. 214-223, 127(4), 2001.

[4] Engelhardt M.O., Rehabilitation strategies for water distribution networks: a literature review with a UK perspective, Urban Water, 2(2), 2000.

[5] Giustolisi, O. \& Berardi, L., Pipe level burst prediction using EPR and MCS-EPR, Water Management Challenges in Global Change, Eds. Ulanicki et al., pp.39-46, Taylor \& Francis, London 2007. 
268 Water Resources Management V

[6] Fantozzi M., Lambert, A., Including the effects of pressure management in calculation of Economic Leakage Level, Proc. of IWA Special Conference Water Loss 2007, Bucharest.

[7] Germanopoulos, G., A technical note on the inclusion of pressure dependent demand and leakage term in water supply network models, Civil Engineering Systems, 2, pp.171-179, 1985. 